Preprint of the paper

"Parallel computing aided design of earthing systems for electrical substations in non homogeneous soil models"

J. Gómez-Calviño, I. Colominas, F. Navarrina, M. Casteleiro, J.M. Cela (2000)

En "Proceedings of the 2000 ICCP Workshops", 381---388; P. Sadayappan (Ed.); IEEE

Computer Society Press, USA. (ISBN: 0-7695-0771-9)

http://caminos.udc.es/gmni 


\section{Parallel computing aided design of earthing systems for electrical substations in non homogeneous soil models}

\author{
J. Gómez-Calviño, I. Colominas, \\ F. Navarrina, M. Casteleiro \\ Applied Mathematics Dpt. \\ Civil Engineering School \\ Universidade da Coruña, SPAIN \\ colominas@iccp.udc.es
}

\author{
J.M. Cela \\ Architecture of Computers Dpt. \\ Universitat Politècnica Catalunya, SPAIN \\ cela@ac.upc.es
}

\begin{abstract}
An accurate design of grounding systems is essential to assure the safety of the persons, to protect the equipment and to avoid interruptions in the power supply. In order to attain these targets, the equivalent electrical resistance of the system and the potential distribution on the earth surface in fault conditions are necessary to compute. In this paper, it is presented a numerical approach for grounding analysis embedded in stratified soils and its implementation in a high-performance parallel computer. The feasibility of this system is shown with its application to the analysis of a real grounding system in a layered soil.
\end{abstract}

\section{Introduction}

The interest for the prediction and the numerical simulation of problems related to the security have increased in last years with the construction of grounding systems of large electrical installations. The "grounding" or "earthing" systems comprises all interconnected grounding facilities of an specific area, being the "grounding grid" the main element of these systems. A grounding grid in most of real electrical substations consists of a mesh of interconnected cylindrical conductors, horizontally buried and supplemented by ground rods vertically thrusted in specific places of the installation site.

The main objective of a grounding system is to provide means to carry and dissipate electrical currents into the ground, in order to guarantee the continuity of the power supply and the integrity of the equipment and to ensure that a person in the vicinity of the grounded installation is not exposed to a critical elec- trical shock. Thus, the apparent electrical resistance of the grounding system must be low enough to guarantee that fault currents dissipate mainly through the earthing electrode into the ground, while the values of electrical potentials between close points on earth surface that can be connected by a person must be kept under certain maximum safe limits (step, touch and mesh voltages) $[1,2]$.

Taking into account these previous aspects, it is obvious that the design of safe grounding systems in electrical installations is essential to assure the security of the persons, to protect the equipment and to avoid interrumptions in the power service. Since the sixties, several methods and procedures for the analysis and design of grounding systems of electrical substations have been proposed, most of them based on practice, on semi-empirical works or on intuitive ideas. Although these techniques represented an important improvement in the grounding analysis area, some problems were reported such as large computational requirements, unrealistic results when segmentation of conductors was increased, and uncertainty in the margin of error $[1,3]$.

Since the early days of the industrial use of the electricity the problem of obtaining the potential distribution produced when a fault current is derived into the ground through an earthing grid has been a challenging one. Although the physical phenomena of fault currents dissipation into the earth is a well-known problem that can be modelled by means of Maxwell's Electromagnetic Theory, its application and resolution for the computing of grounding grids of large installations in practical cases present some difficulties. First, it is obvious that no analytical solutions can be obtained in a real case. Moreover, the specific geometry of the grounding systems (a mesh 
of interconnected bare conductors in which ratio diameter/length is relatively small) precludes the use of standard numerical techniques (such as the Finite Element Method or Finite Differences) since discretization of the domain (the whole ground) is required, and obtaining sufficiently accurate results should imply unacceptable computing efforts in memory storage and CPU time. For these reasons, during the last years the authors have developed a general numerical formulation based on the Boundary Element Method for the analysis of earthing systems embedded in uniform soil models, which has been succesfully applied to real grounding grids $[4,5]$. At present, for real problems, single-layer models ("uniform models") run in real-time in personal computers[6], while multiple-layer models break off the design process since the computing time is not contemptible.

Next, we present a generalization of the BEM formulation for the analysis of grounding systems embedded in stratified soils and the study of the parallelization of that code for its implementation in a high-performance parallel computer. Furthermore, we apply this approach to the analysis of a real grounding system in a layered soil model.

\section{Mathematical Model of the Problem}

Fault current dissipation into the ground through a grounding grid can be described by means of Maxwell's Electromagnetic Theory. Thus, if one restricts the analysis to the electrokinetic steady-state response and neglects the inner resistivity of the earthing conductors (then, potential can be assumed constant in every point of the grounding electrode surface), the $3 \mathrm{D}$ problem can be written as

$$
\begin{gathered}
\operatorname{div}(\boldsymbol{\sigma})=0, \quad \boldsymbol{\sigma}=-\boldsymbol{\gamma} \operatorname{grad}(V) \text { in } E ; \\
\boldsymbol{\sigma}^{t} \boldsymbol{n}_{E}=0 \text { in } \Gamma_{E} ; \quad V=V_{\Gamma} \text { in } \Gamma ; \\
V \rightarrow 0, \text { if }|\boldsymbol{x}| \rightarrow \infty ;
\end{gathered}
$$

where $E$ is the earth, $\boldsymbol{\gamma}$ is its conductivity tensor, $\Gamma_{E}$ is the earth surface, $\boldsymbol{n}_{E}$ is its normal exterior unit field and $\Gamma$ is the electrode surface [5]. Therefore, when the earthing electrode is energized to a voltage $V_{\Gamma}$ (Ground Potential Rise, or GPR) relative to a remote earth, the solution to problem (1) gives potential $V$ and current density $\boldsymbol{\sigma}$ at an arbitrary point $\boldsymbol{x}$. Then, for known values of $V$ on $\Gamma_{E}$ and $\boldsymbol{\sigma}$ on $\Gamma$, it is straightforward to obtain the design and safety parameters of the grounding system, such as the total surge current, the equivalent resistance of the grounding system, and the step and touch voltages[1,2]. On the other hand, since $V$ and $\boldsymbol{\sigma}$ are proportional to the GPR value, it will be used the normalized boundary condition $V_{\Gamma}=1$ from here on.
Most of the methods proposed for grounding analysis are based on the hypothesis that soil can be considered homogeneous and isotropic, and thus $\boldsymbol{\gamma}$ is substituted by an apparent scalar conductivity $\gamma$ that must be experimentally obtained[1]. It is obvious that this assumption does not introduce significant errors if the soil is essentially uniform (both in horizontal and vertical directions) in the surroundings of the grounding $\operatorname{grid}[1]$, and this model can be used with loss of accuracy if the soil resistivity changes slightly with depth. However, since parameters involved in the grounding design can significantly vary if soil electrical properties change through the substation site (e.g., changes of the material nature, changes of humidity), it seems advisable to develop advanced models that could take into account variations of soil conductivity in the surroundings of the installation.

It is clear that to take into account the real variation of the soil conductivity in the vicinity of a grounding site would never be affordable, neither from the economical nor from the technical point of view. Hence, a more practical proposed soil model (and still quite realistic when conductivity is not markedly uniform with depth) consists of considering the soil stratified in a number of horizontal layers, defined by an appropriate thickness and an apparent scalar conductivity that must be experimentally obtained. In fact, it is widely accepted that two-layer (or even three-layer) soil models should be sufficient to obtain good and safe designs of grounding systems in most practical cases[1]. This paper is devoted to studying the application of high performance computing techniques to two-layer soil models by means of parallelization, and its final implementation.

Consequently, if the soil is formed by $C$ horizontal layers with different conductivities and the grounding electrode is buried in the layer $b$, the mathematical problem (1) can be written in terms of the following Neumann exterior problem[7]

$$
\begin{aligned}
\operatorname{div}\left(\boldsymbol{\sigma}_{c}\right)= & 0, \boldsymbol{\sigma}_{c}=-\gamma_{c} \operatorname{grad}\left(V_{c}\right) \text { in } E_{c}, 1 \leq c \leq C ; \\
& \boldsymbol{\sigma}_{1}^{t} \boldsymbol{n}_{E}=0 \text { in } \Gamma_{E}, V_{b}=1 \text { in } \Gamma \\
V_{c} \rightarrow & 0 \text { if }|\boldsymbol{x}| \rightarrow \infty, \boldsymbol{\sigma}_{c}^{t} \boldsymbol{n}_{c}=\boldsymbol{\sigma}_{c+1}^{t} \boldsymbol{n}_{c} \text { in } \Gamma_{c} \\
& 1 \leq c \leq C-1 ;
\end{aligned}
$$

being $E_{c}$ each one of the soil layers, $\gamma_{c}$ the scalar conductivity of layer $E_{c}, V_{c}$ the potential at an arbitrary point of layer $E_{c}$ and $\boldsymbol{\sigma}_{c}$ the corresponding current density, $\Gamma_{c}$ the interface between layers $E_{c}$ and $E_{c+1}$ and $\boldsymbol{n}_{c}$ the normal field to $\Gamma_{c}[7]$. 


\section{Variational Form of the BVP}

As we have exposed, the real geometry of grounding systems in most of real electrical substations consists of a grid of interconnected bare cylindrical conductors, horizontally buried and supplemented by rods, so ratio diameter/length uses to be relatively small $\left(\sim 10^{-3}\right)$.

Although this geometry is straightforward to describe, it implies serious difficulties to the modellization of the problem: it is obvious that no analytical solutions can be obtained in a real case, and the use of standard numerical techniques (FEM or FD) should involve a completely out of range computing effort since discretization of the domain (the $3 \mathrm{D}$ domains $E_{c}$ ) is required. Consequently, and taking into account that potential values are only required on the earth surface and the equivalent resistance can be easily obtained in terms of the leakage current density $\sigma$ on $\Gamma\left(\sigma=\boldsymbol{\sigma}^{t} \boldsymbol{n}\right.$, where $\boldsymbol{n}$ is the normal exterior unit field to $\Gamma$ ), we turn our attention to a Boundary Element approach which would only require the discretization of the grounding surface $\Gamma[5]$. For this, it is first necessary to derive an expression to relate the potential $V$ and the leakage current density $\sigma$.

Since the surroundings of the substation site are levelled and regularized during the construction of the electrical installation, earth surface $\Gamma_{E}$ and interfaces $\Gamma_{c}$ between layers can be assumed horizontal. Thus, with this assumption the application of the "method of images" and Green's Identity to problem (2) yields the following integral expression[7] for potential $V_{c}\left(\boldsymbol{x}_{c}\right)$ at an arbitrary point $\boldsymbol{x}_{c} \in E_{c}$, in terms of the unknown leakage current density $\sigma(\boldsymbol{\xi})$ at any point $\boldsymbol{\xi}$ of the electrode surface $\Gamma \subset E_{b}$ :

$$
V_{c}\left(\boldsymbol{x}_{\boldsymbol{c}}\right)=\frac{1}{4 \pi \gamma_{b}} \iint_{\boldsymbol{\xi} \in \Gamma} k_{b c}\left(\boldsymbol{x}_{c}, \boldsymbol{\xi}\right) \sigma(\boldsymbol{\xi}) d \Gamma, \forall \boldsymbol{x}_{c} \in E_{c},
$$

where integral kernels $k_{b c}\left(\boldsymbol{x}_{c}, \boldsymbol{\xi}\right)$ are formed by infinite series of terms corresponding to the resultant images obtained when Neumann exterior problem (2) is transformed into a Dirichlet one[7, 9]. In the case of uniform soil, the series are reduced to only two summands, since there is only one image of the original $\operatorname{grid}[4,5]$.

Weakly singular kernel $k_{b c}\left(\boldsymbol{x}_{c}, \boldsymbol{\xi}\right)$ depends on the conductivity of the layers, and on the inverse of the distances from the point $\boldsymbol{x}_{c}$ to the point $\boldsymbol{\xi}$ and to all the images of $\boldsymbol{\xi}$ with respect to the earth surface $\Gamma_{E}$ and to the interfaces $\Gamma_{c}$ between layers[7]. A general form to express these integral kernels is given by:

$$
k_{b c}\left(\boldsymbol{x}_{c}, \boldsymbol{\xi}\right)=\sum_{l=0}^{\infty} k_{b c}^{l}\left(\boldsymbol{x}_{c}, \boldsymbol{\xi}\right), \quad k_{b c}^{l}\left(\boldsymbol{x}_{c}, \boldsymbol{\xi}\right)=\frac{\psi^{l}(\kappa)}{r\left(\boldsymbol{x}_{c}, \boldsymbol{\xi}^{l}(\boldsymbol{\xi})\right)},
$$

being $\psi^{l}$ a weighting coefficient that only depends on a certain ratio $\kappa$ defined in terms of the layer conductivities and $r\left(\boldsymbol{x}_{c}, \boldsymbol{\xi}^{l}(\boldsymbol{\xi})\right)$ the Euclidean distance between the points $\boldsymbol{x}_{c}$ and $\boldsymbol{\xi}^{l}$, being $\boldsymbol{\xi}^{0}$ the point $\boldsymbol{\xi}$ on the electrode surface $\left(\boldsymbol{\xi}^{0}(\boldsymbol{\xi})=\boldsymbol{\xi}\right)$, where $\boldsymbol{\xi}^{l}(l \neq 0)$ are the images of $\boldsymbol{\xi}$ with respect to the earth surface and to the interfaces between layers[7]. For example, in the particular case of a two-layer soil model ratio $\kappa$ is given by $\left(\gamma_{1}-\gamma_{2}\right) /\left(\gamma_{1}+\gamma_{2}\right)$ and four cases must be considered depending on the layer where the points $\boldsymbol{x}_{c}$ and $\boldsymbol{\xi}^{l}$ are located. In each case, several series with different locations of their images and different weighting coefficients are added. Explicit expressions of these kernels can be found in $[7,9]$.

Now, since the expression for the potential (3) also holds on electrode surface $\Gamma$ (where potential is known by the boundary condition $V_{b}(\boldsymbol{\chi})=1, \forall \boldsymbol{\chi} \in \Gamma$ ), the leakage current density $\sigma$ must satisfy the following Fredholm integral equation of the first kind on $\Gamma$ :

$$
\iint_{\boldsymbol{\chi} \in \Gamma} w(\boldsymbol{\chi})\left(\frac{1}{4 \pi \gamma_{b}} \iint_{\boldsymbol{\xi} \in \Gamma} k_{b b}(\boldsymbol{\chi}, \boldsymbol{\xi}) \sigma(\boldsymbol{\xi}) d \Gamma-1\right) d \Gamma=0
$$

for all members $w(\boldsymbol{\chi})$ of a suitable class of test functions defined on $\Gamma[4,5]$. It is obvious that a numerical approach based on the Boundary Element Method seems to be the best choice to solve equation (5).

\section{BEM Numerical Formulation}

\subsection{General 2D approach}

The numerical resolution of the variational form (5) requires the discretization of the domain (the surface of the cylindrical conductors) and the leakage current density that flows from the grounded electrode. Thus, for given sets of $\mathcal{N}$ trial functions $\left\{N_{i}(\boldsymbol{\xi})\right\}$ defined on $\Gamma$, and $\mathcal{M}$ two dimensional boundary elements $\left\{\Gamma^{\alpha}\right\}$, the leakage current density $\sigma$ and the electrode surface $\Gamma$ can be discretized as follows:

$$
\sigma(\boldsymbol{\xi})=\sum_{i=1}^{\mathcal{N}} \sigma_{i} N_{i}(\boldsymbol{\xi}), \quad \Gamma=\bigcup_{\alpha=1}^{\mathcal{M}} \Gamma^{\alpha},
$$

Now, if we take into account that kernels (4) are given by series, integral expression (3) for potential $V_{c}\left(\boldsymbol{x}_{c}\right)$ can also be discretized as

$$
\begin{gathered}
V_{c}\left(\boldsymbol{x}_{c}\right)=\sum_{i=1}^{\mathcal{N}} \sigma_{i} V_{c, i}\left(\boldsymbol{x}_{c}\right) ; V_{c, i}\left(\boldsymbol{x}_{c}\right)=\sum_{\alpha=1}^{\mathcal{M}} \sum_{l=0}^{l_{V}} V_{c, i}^{\alpha l}\left(\boldsymbol{x}_{c}\right) \\
V_{c, i}^{\alpha l}\left(\boldsymbol{x}_{c}\right)=\frac{1}{4 \pi \gamma_{b}} \iint_{\boldsymbol{\xi} \in \Gamma^{\alpha}} k_{b c}^{l}\left(\boldsymbol{x}_{c}, \boldsymbol{\xi}\right) N_{i}(\boldsymbol{\xi}) d \Gamma^{\alpha} ;
\end{gathered}
$$


where $l_{V}$ represents the number of terms that is necessary to consider until convergence is achieved.

On the other hand, for a given set of $\mathcal{N}$ test functions $\left\{w_{j}(\boldsymbol{\chi})\right\}$ defined on $\Gamma$, variational form (5) is reduced to the following linear system:

$$
\begin{gathered}
\sum_{i=1}^{\mathcal{N}} R_{j i} \sigma_{i}=\nu_{j} \quad(j=1, \ldots, \mathcal{N}) \\
R_{j i}=\sum_{\beta=1}^{\mathcal{M}} \sum_{\alpha=1}^{\mathcal{M}} \sum_{l=0}^{l_{R}} R_{j i}^{\beta \alpha l}, \quad \nu_{j}=\sum_{\beta=1}^{\mathcal{M}} \nu_{j}^{\beta},
\end{gathered}
$$

being coefficients $R_{j i}^{\beta \alpha l}$ and $\nu_{j}^{\beta}$ :

$$
\begin{gathered}
R_{j i}^{\beta \alpha l}=\frac{1}{4 \pi \gamma_{b}} \iint_{\boldsymbol{\chi} \in \Gamma^{\beta}} w_{j}(\boldsymbol{\chi}) \iint_{\boldsymbol{\xi} \in \Gamma^{\alpha}} k_{b b}^{l}(\boldsymbol{\chi}, \boldsymbol{\xi}) N_{i}(\boldsymbol{\xi}) d \Gamma^{\alpha} d \Gamma^{\beta} \\
\nu_{j}^{\beta}=\iint_{\boldsymbol{\chi} \in \Gamma^{\beta}} w_{j}(\boldsymbol{\chi}) d \Gamma^{\beta}
\end{gathered}
$$

where $l_{R}$ represents the number of terms that is necessary to consider until convergence is achieved.

It is important to emphasize that the solution of linear equations (9) provides the values of the intensities $\sigma_{i}(i=1, \ldots, \mathcal{N})$ leaking from the nodes of the grid. With these values, it is possible to compute the potential at any point on the earth surface or even at any inner point - by means of (7) and (8) - , the leakage current density $\sigma$ - by means of (6)-, and all the design and safety parameters of the grounding system $[1,5]$.

Nevertheless, the statement of the above linear system requires the discretization of a $2 \mathrm{D}$ domain: the whole surface $\Gamma$ of the grounding electrodes, which implies a large number of degrees of freedom in practical cases. Besides, its matrix is full and the computation of its coefficients requires to perform double integration on $2 \mathrm{D}$ domains. For all these reasons, it is necessary to introduce some additional hypotheses in order to decrease the computational cost.

\subsection{Approximated 1D boundary element approach}

An approximated boundary element numerical approach to the previous one presented can be derived if the real geometry of the grounding systems in practice is considered. Thus, since the ratio between the diameter and the length of the grounded conductors is very small $\left(\sim 10^{-3}\right)$, the hypothesis of circumferential uniformity (i.e., the leakage current density $\sigma$ is constant around the cross section of the cylindrical conductors of the grounding grid) can be assumed producing a notable fall of the computational cost $[1,5]$. In this way, discretizations (6) and (9) become much simpler, since the classes of test and trial functions are restricted to those with circumferential uniformity, while only the axial lines of the grounding electrodes have to be discretized[5].

Now, for a given level of mesh refinement, the number of element contributions $R_{j i}^{\beta \alpha}$ and $\nu_{j}^{\beta}$ that we need to compute in order to state linear system (9), as much as the number of unknowns $\sigma_{i}$ are significantly smaller than in the previous $2 \mathrm{D}$ approach, since it is required the discretization of a simpler domain: the axial lines of the grounding electrodes. Despite of this important reduction in the computation effort, extensive computing is still necessary mainly because of the circumferential integration on the perimeter of the electrodes that are involved in the integral kernels[5]. However, these circumferential integrals can be performed in an approximated way if suitable simplifications in the general approach are introduced[7].

The selection of different sets of trial and test functions in the numerical scheme allows to derive different formulations. Further discussion in this paper is restricted to the case of a Galerkin type approach, since the matrix of coefficients is symmetric and positive definite[5]. As the 1D approximated expressions for terms $V_{c, i}^{\alpha l}$ and $R_{j i}^{\beta \alpha l}$ in (8) and (10) are formally equivalent to those obtained in the case of uniform soil models[7], these terms can be computed by using the highly efficient analytical integration techniques derived by the authors to compute these coefficients for the uniform soil case[5, 7].

The example presented in the next section corresponds to the analysis of a grounding system embedded in a two-layer soil model. Obviously, this boundary element formulation can be applied to any other case with a higher number of layers. However, CPU time may increase up to unadmisible levels, mainly due to the poor rate of convergence of the underlying series expansions, and the need to evaluate double series (in three-layer models), triple series (in four-layer models), and so on.

\subsection{Total Efficiency of the Numerical Ap- proach}

An important aspect of the numerical formulation proposed is its total computational cost. Thus, for specific discretization $(\mathcal{M}$ elements of $p$ nodes each, and a total number of $\mathcal{N}$ degrees of freedom), a linear system (9) of order $\mathcal{N}$ must be generated and solved.

Matrix generation process requires $O\left(\mathcal{M}^{2} p^{2} / 2\right)$ operations, since $p^{2}$ series of contributions of type (10) have to be computed for every pair of elements, and ap- 
proximately half of them are discarded because of symmetry. In uniform soil models these series are formed by only two terms, while in two-layer models the series have an infinite number of them, that will be numerically added up until a tolerance is fulfilled or an upper limit of summands is achieved. Consequently, matrix generation will be much more expensive in two-layer models. In connection with the linear system solving process, it requires $O\left(\mathcal{N}^{3} / 3\right)$ operations (since the matrix is symmetric but not sparse) if the resolution is carried out with a direct method.

Hence, most of computing effort is devoted to matrix generation in small/medium problems, while linear system resolution prevails in medium/large ones. In these cases, the use of direct methods for the linear system resolution is out of range. Therefore iterative or semiiterative techniques will be preferable. The best results have been obtained by a diagonal preconditioned conjugate gradient algorithm with assembly of the global matrix[5]. This technique has turned out to be extremely efficient for solving large scale problems, with a very low computational cost in comparison with matrix generation. So the cost of the system resolution should never prevail.

On the other hand, once the leakage current has been obtained, the cost of computing the equivalent resistance is negligible. The additional cost of computing potential at any given point (normally at the earth surface) by means of $(7)$ only requires $O(\mathcal{M p})$ operations, since $p$ series of contributions of type (8) have to be computed for every element. However, if it is necessary to compute potentials at a large number of points (i.e. to draw contours), computing time may be important.

\section{Parallelization of the BEM approach and application to a practical case}

\subsection{Description of the earthing system}

This boundary element approach has been integrated in a Computer Aided Design system for grounding analysis. In this section we will discuss the implementation of the numerical code and its parallelization, that is, the distribution of different tasks of the program among several processors. Next discussion will be illustrated with its application to a real case, in order to test the parallel code onto a large grid: the Barberá substation, near the city of Barcelona in Spain. This earthing system is formed by a grid of 408 segments of cylindrical conductor of the same diameter $(12.85$ $\mathrm{mm}$ ) buried to a depth of $80 \mathrm{~cm}$. The grounding system has a right-angled triangle shape of $143 \times 89 \mathrm{~m}$ and

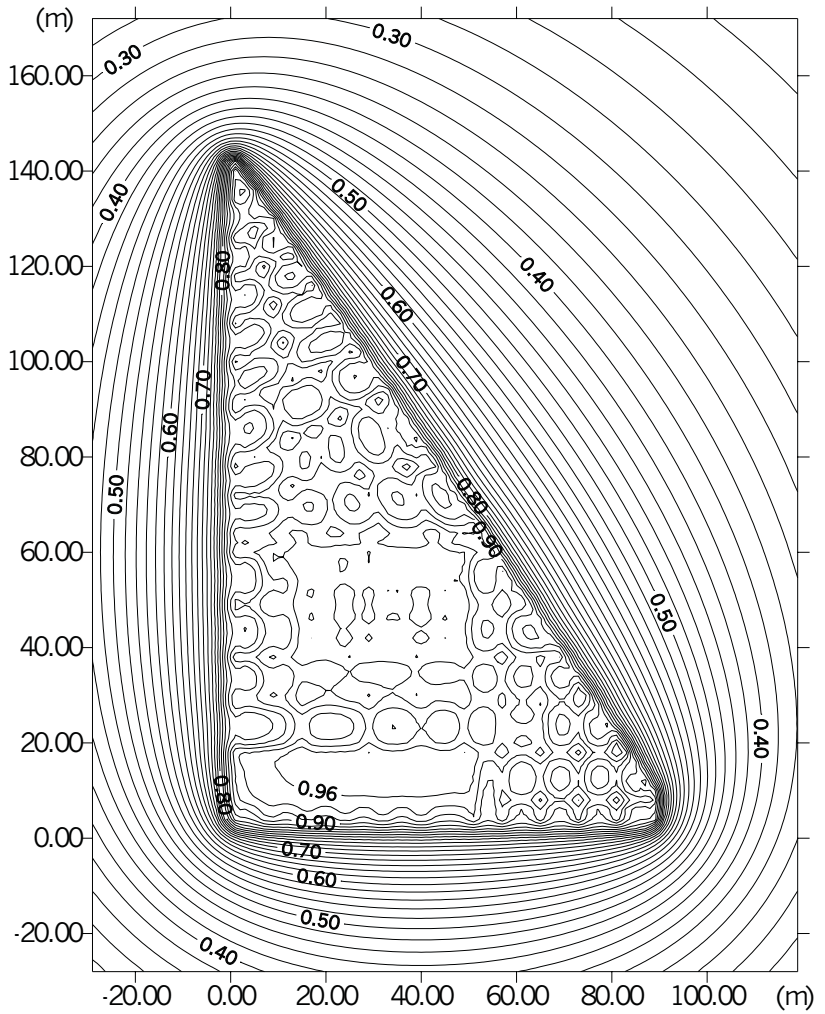

Figure 1. Barberá grounding system: Potential distribution on earth surface $(\times 10 \mathrm{kV})$ obtained by using a uniform soil model.

protects a total area of $6,600 \mathrm{~m}^{2}$. The grid has been discretized in 408 linear leakage current elements which implies 238 degrees of freedom. The Ground Potential Rise (GPR) considered in this study has been $10 \mathrm{kV}$.

This grounding system has been calculated by using a uniform soil model $\left(\gamma=0.016(\Omega m)^{-1}\right)$, and a more interesting two-layer soil model (the conductivities of the upper and the lower layer are $\gamma_{1}=0.005(\Omega m)^{-1}$ and $\gamma_{2}=0.016(\Omega m)^{-1}$ respectively, and the thickness of the upper layer is $1.0 \mathrm{~m}$ ).

Figure 1 shows the potential distribution on the earth surface obtained for the uniform soil case, and Figure 2 does for the two-layer soil model. The equivalent resistance and the total surge current of the earthing system computed in each case has been 0.3128 $\Omega$ and $\mathrm{I}=31.97 \mathrm{kA}$ (for the uniform soil model), and $0.3704 \Omega$ and $\mathrm{I}=26.99 \mathrm{kA}$ (for the two-layer soil model).

As we can see in this example, results obtained by using a multiple-layer soil model can be noticeably different from those obtained by using a single layer (or uniform) soil model. Therefore, it could be advisable to 


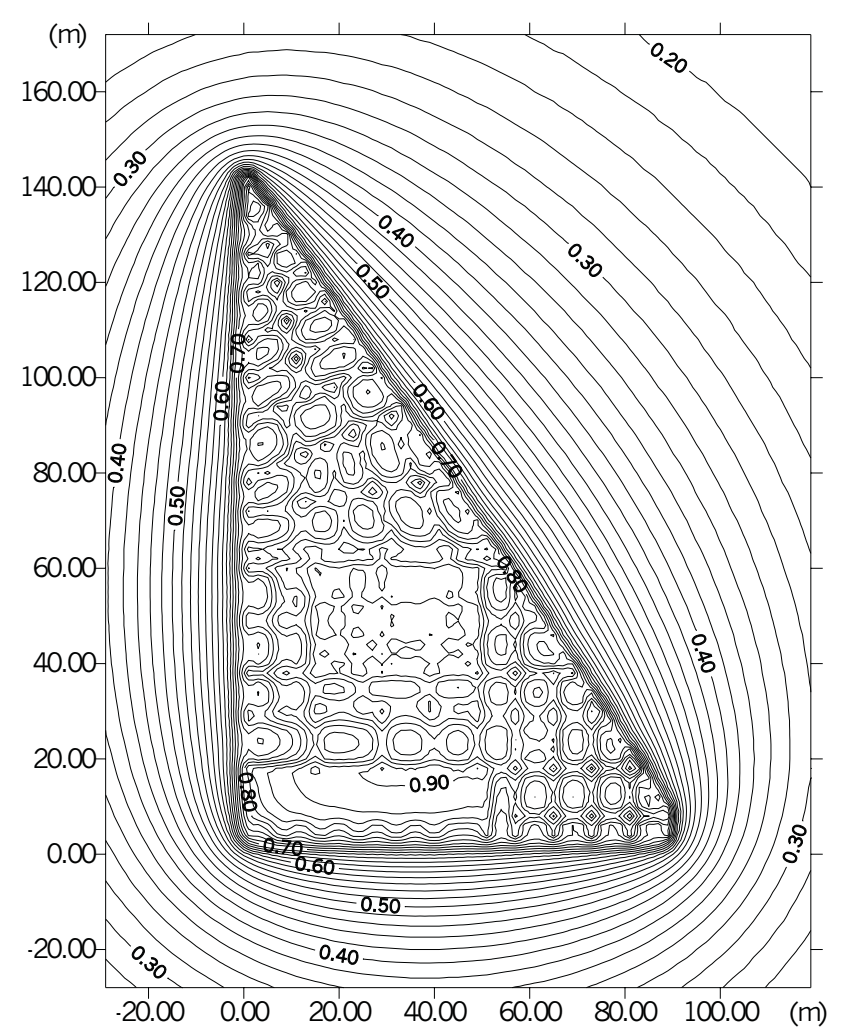

Figure 2. Barberá grounding system: Potential distribution on earth surface $(\times 10 \mathrm{kV})$ obtained by using a two-layer soil model.

use multi-layer soil formulations to analyze grounding systems as a general rule, in spite of the increase of the computational effort. In fact, the use of this kind of advanced models should be mandatory in cases where the conductivity of the soil changes markedly with depth.

However, while single-layer models run in real time in conventional computers for the analysis of medium/big size grounding grids, multiple-layer models require in general an out of order computing time. For this reason, we have studied the parallelization of the multi-layer boundary element numerical approach which could become a real-time design tool for grounding analysis.

\subsection{Parallelization of the numerical appro- ach}

As we have exposed in previous sections, the most critical time-consuming process of this numerical formulation based on the Boundary Element Method is matrix generation, followed by computation of potential at a large number of points once the leakage current
Table 1. Barberá Grounding System: CPU time in sequential execution for each process of the numerical approach

\begin{tabular}{|lr|}
\hline Process & \multicolumn{1}{c|}{ Time(s) } \\
\hline Data Input: & 0.737 \\
Data Preprocessing: & 0.045 \\
Matrix Generation: & 1723.207 \\
Linear System Solving: & 0.211 \\
Resuts Storage: & 0.015 \\
\hline
\end{tabular}

density has been obtained. Since both processes accept massive parallelization, computing time could be reduced under acceptable levels, even for cases of extremely large models, if the number of available processors is high enough, in spite of the efficiency loses due to the data transfer overhead and the system administration workload. Table 1 summarizes the CPU time required in each process of the implementation of the numerical approach for the Barberá grounding system in the two-layer soil model case. It is evident that the matrix construction is the most expensive part of it.

The numerical approach has been implemented on a CAD system, which has been compiled and run onto an Origin 2000 Silicon Graphics computer at the European Center for Parallelism of Barcelona (CEPBA). The compilation process of the code has been made in sequential and parallel modes, and the executions have been run for the uniform and the two-layer models.

The O2000 used in our work is a high-performance computer with 64 MIPS R10000 processors at 250 $\mathrm{MHz}$. It has a peak performance of 32 GFlops. Internally, the $\mathrm{O} 2000$ is organised in clusters of 2 processors sharing a main memory of 256 Mbyte. Each processor has 4 Mbyte of cache memory. The clusters are connected by an hypercube network. Each processor can access all the distributed main memory through the network. Then, the O2000 can be programmed as an 8 Gbyte shared memory machine. The input/output devices have a capability of 1.2 Gbytes/s.

The parallelization mode selected for this problem has been the use of compiler directives, following the present OpenMP standard. This selection is justified because: a) a shared memory computer is available for running the program (necessary condition for using compiler parallelization directives), b) the use of compiler directives grants clearness to a parallel code that may be handled in the future, c) the OpenMP syntax assures the portability of the parallel code to any shared memory computer, and d), as we will see below, the loop to be run in parallel is transformable into an adequate form so that directives are efficient. 
In the sequential program, the matrix generation process is performed by means of a double loop that couples every element with all the other ones $(\mathcal{M}(\mathcal{M}+$ $1) / 2$ cycles). Into each cycle, the elemental matrix corresponding to a pair of elements is calculated and immediately assembled into the system matrix. If we try to parallelize this double loop, we find that the assembly of the elemental matrices causes a dependency between the actions of the threads or processes. This drawback can be avoided by taking the assembly process out of that loop, which implies first the computation and the storage of all the elemental matrices and, after this step, the assembly in a sequential mode. This scheme requires approximately twice memory space than the original one, but in any case this memory space is not very large. If $\mathcal{N}=O\left(10^{3}\right)$ then the matrix size is $O\left(10^{6}\right)$ bytes.

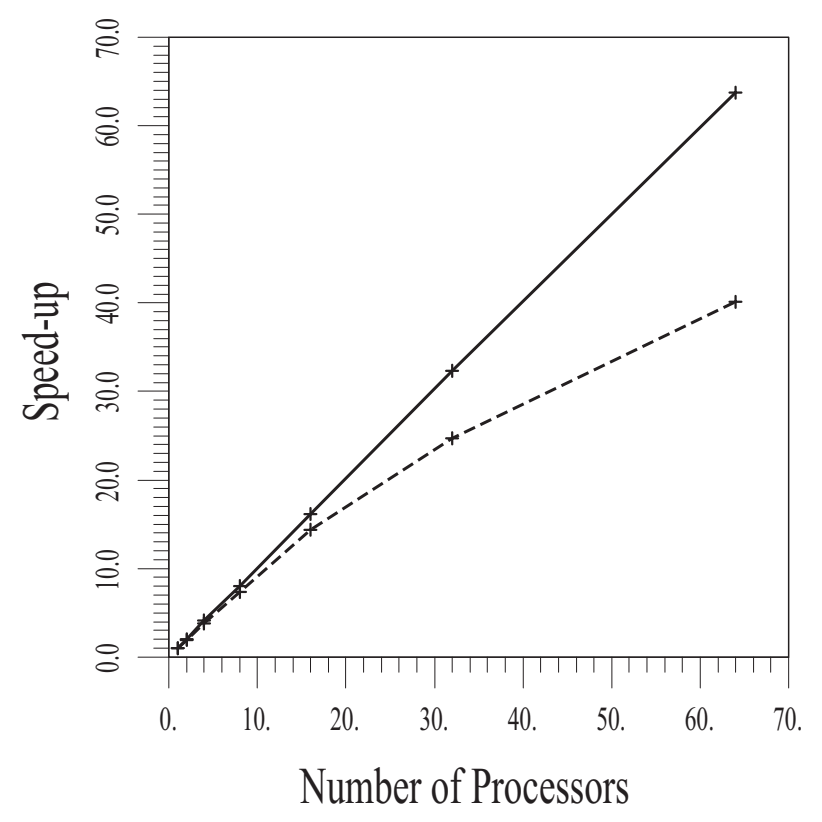

Figure 3. Barberá grounding system: Comparision between the speed-up obtained with the parallelization of the outer loop (in continuous line) and the inner loop (in discontinuous line).

Then, our target code is the nesteed DO loops that compute the elemental matrices, and we can parallelize the outer loop or the inner one. This is the first choice studied. Figure 3 shows the evolution of the speedup factor obtained with different number of processors for both types of parallelization in the analysis of the Barberá grounding system with a two-layer soil model (the speed-up factor has been referenced to the sequential CPU time). These results have been obtained with
Table 2. Barberá Grounding System: Speed-up factors for different schedules and number of processors in the outer loop parallelization

\begin{tabular}{|l|r|r|r|r|}
\hline \multirow{2}{*}{ Schedule () } & \multicolumn{4}{|c|}{ Number of Processors } \\
\cline { 2 - 5 } & 1 & 2 & 4 & 8 \\
\hline Static & 1.01 & 1.32 & 2.32 & 4.38 \\
Static,64 & 1.02 & 1.76 & 1.86 & 3.55 \\
Static,16 & 1.02 & 1.94 & 3.59 & 6.23 \\
Static, 4 & 1.01 & 2.01 & 3.96 & 7.36 \\
Static, 1 & 1.02 & 2.03 & 4.03 & 7.99 \\
Dynamic,64 & 1.02 & 2.02 & 3.56 & 3.55 \\
Dynamic,16 & 1.02 & 2.02 & 4.08 & 7.87 \\
Dynamic, 4 & 1.01 & 2.04 & 3.99 & 7.90 \\
Dynamic, 1 & 1.02 & 2.03 & 4.09 & 8.05 \\
Guided,64 & 1.02 & 1.97 & 3.56 & 3.56 \\
Guided,16 & 1.02 & 1.99 & 3.96 & 8.03 \\
Guided, 4 & 1.02 & 2.01 & 4.11 & 7.93 \\
Guided, 1 & 1.02 & 2.07 & 3.95 & 8.38 \\
\hline
\end{tabular}

the schedule option "Dynamic, 1 " (we will discuss the schedule options below), and they correspond to the minimum of $4 \mathrm{CPU}$ time measures made for the same option (the variance of the four ones is very small, anyway), in order to approximate better the strict value. In some cases, we have obtained speed-ups bigger than the number of processors due to small errors in the measurement of CPU time by the processors, and to the additional optimization of the code that the parallel compiler introduces.

Results are better when the outer loop is parallelized because the granularity is bigger in that way, and so the cost of managing the parallel execution is minor: since the numerical approach leads to a symmetric formulation, the coupling of every element of the grid with each one of the others can be represented by a triangle of $\mathcal{M}$ columns, of which the first one has $\mathcal{M}$ rows and the last one has 1 row. Hence, if the outer loop is parallelized, the columns of the triangle, that is, the cycles of the outer loop, are distributed among the processors. Whereas, if the inner loop is parallelized, the rows of one column are distributed among the processors. In this case, when computations on that column are finished the program moves sequentially to the next one, where another distribution of its rows among the processors is performed. This effect of granularity is, of course, more sensible when the number of processors grows, as figure 3 shows.

As the parallelization of the outer loop is preferable, and the cycles that it is necessary to distribute among the processors have very different sizes, the way to do 
this distribution becomes a decisive question. Table 2 summarizes the speed-up factors (referenced to the sequential CPU time) obtained for the outer-loop parallelization with different number of processors by using different "schedule" OpenMP options. Since the size of the cycles is linearly decreasing, "static" schedules with a high "chunk" (i.e., the number of cycles in a task) are the less profitable ones. When no chunk value is specified, all the columns are uniformly distributed in the beginning. "Dynamic" schedules improve this behaviour because as each processor finishes a task, it dynamically takes the next one. Best results are obtained for a dynamic schedule with a chunk parameter of 1 column. This is the most lively scheme, since there are never waiting processors, although it requires the biggest amount of parallelization management. "Guided" schedules distribute initially all the columns among all the processors into pieces with size exponentially varying. In this case, results are very similar to those obtained with the "dynamic" ones. In general, for any schedule, we obtained worse results when the chunk parameter and the number of processors are high because then some processors do not get any work.

Therefore, as we can see, speed-up factors obtained for the outer parallelization are very close to the number of processors for good schedules, that is, "dynamic" or "guided" with low chunk parameters. This fact reveales that the parallelization of this loop is very profitable: in the example, the Barberá grounding analysis in a two layer soil model, $99.9 \%$ of the work of the whole processing program is dealt with only 408 tasks.

\section{Conclusions}

Accurate analysis of grounding systems of electrical instalations requires the use of multilayer soil models when the soil is not essentially uniform in the surroundings of the earthing grid. At present, while uniform soil models run in real-time in conventional computers, the use of models with a small number of soil layers breaks off the design process due to the important computing time required in practical cases.

In last years, the authors have developed a BEM numerical approach for the analysis of grounding systems embedded in stratified soils that has been implemented in a high performance parallel computer. This numerical approach accepts massive parallelization since most of the computing time is required in the computation of the elemental matrices corresponding to the contribution of each electrode of the grounding grid. Results obtained with different parallelization options prove that the proposed multi-layer Boundary
Element formulation can be a real-time design tool, as high-performance parallel computing becomes a widespread available resource in engineering.

\section{Acknowledgements}

This work has been partially financed by the project 1FD97-0108 of the Ministerio de Educación y Cultura, SGPICYT (cofinanced with FEDER European Union funds and the power company UFISA), and by research fellowships of Xunta de Galicia, CEPBA and Universidade da Coruña.

\section{References}

[1] ANSI/IEEE Std.80, IEEE Guide for safety in AC substation grounding, New York, 1986.

[2] Sverak J.G., Progress in Step and Touch Voltage Equations of ANSI/IEEE Std.80, IEEE Trans. Power Delivery,13, 762-767, 1999.

[3] Garret D.L., Pruitt J.G., Problems Encountered with the APM of Analyzing Grounding Systems, IEEE Trans. P.A.S.,104, 4006-4023, 1985.

[4] Navarrina F., Colominas I., Casteleiro M., Analytical Integration Techniques for Earthing Grid Computation by BEM, Num. Met. in Eng. and Appl. Sci., 1197-1206, CIMNE, Barcelona, 1992.

[5] Colominas I., Navarrina F., Casteleiro M., A boundary element numerical approach for grounding grid computation, Comput. Methods Appl. Mech. Engrg., 174, 73-90, 1999.

[6] M. Casteleiro, L.A. Hernández, I. Colominas and F. Navarrina, Memory and User guide of system TOTBEM for CAD of grounding grids in electrical installations, (in spanish), Civil Engrg. Sch., Universidad de La Coruña, 1994.

[7] Colominas I., Navarrina F., Casteleiro M., A Numerical Formulation for Grounding Analysis in Stratified Soils, Submitted to IEEE Trans. Power Delivery, 1999.

[8] Colominas I., Navarrina F., Mosqueira G., Eiroa J.M., Casteleiro M., Numerical modelling for grounding grids in high-performance parallel computers, International Series on Advances in Boundary Elements: Boundary Elements XXII, Comp. Mech. Pub., Southampton, UK, 2000.

[9] G.F. Tagg, Earth Resistances, Pitman Pub. Co., New York, 1964. 